\title{
Assimilating and Modeling Dust Transport in the Martian Climate System
}

\author{
Tao Ruan ${ }^{1}$, Luca Montabone ${ }^{1,3}$, Peter L. Read ${ }^{1}$ \\ and Stephen R. Lewis ${ }^{2}$ \\ ${ }^{1}$ Atmospheric, Oceanic \& Planetary Physics, University of Oxford, \\ Oxford, OX1 3PU, United Kingdom \\ email: ruan@atm.ox.ac.uk \\ ${ }^{2}$ Dept. of Physical Sciences, \& The Open University, \\ Walton Hall, Milton Keynes, MK7 6AA, United Kingdom \\ email: s.r.lewis@open.ac.uk \\ ${ }^{3}$ Université Pierre et Marie Curie, Laboratoire de Météorologie Synamique, \\ Paris, France \\ email: montabone@atm.ox.ac.uk
}

\begin{abstract}
A meteorological data assimilation system has been developed recently for analyzing measurements of temperature and dust opacity on Mars and has been successfully applied in several studies (e.g. Montabone et al. 2005, Lewis et al. 2007) to study various atmospheric phenomena. A more sophisticated data assimilation system, now with full dust transport incorporated, is becoming available to represent more accurately and realistically the physical transport of dust.
\end{abstract}

Keywords. hydrodynamics, planets and satellites: general, methods: data analysis, methods: numerical

\section{Overview}

With the rapidly expanding discoveries of extra-solar planets around a wide variety of nearby stars, it is more important than ever to extend and develop our understanding of the evolution, structure and climate of the planets within our own Solar System. In this context, we have recently combined a Martian Global Circulation Model (MGCM), with a meteorological data assimilation scheme to analyze the retrievals from the Thermal Emission Spectrometer (TES) on board NASA's Mars Global Surveyor (MGS) (Smith 2004) and from the Mars Climate Sounder (MCS) on board Mars Reconnaissance Orbiter (MRO) (McCleese et al. 2007). This approach is able to provide a complete fourdimensional solution consistent with both observations and with physical constraints and balances represented by a numerical model.

The MGCM we use combines a spectral dynamical solver and a tracer transport scheme, developed in the UK, and a physics package developed jointly with Laboratoire de Météorologie Dynamique (Paris, France) and Instituto de Astrofisica de Andalucia (Granada, Spain), including the representations of radiative transfer, planetary boundary layer physics, dust lifting, etc. The data assimilation scheme implemented in the MGCM is computationally inexpensive compared to the MGCM itself, and based on the Analysis Correction sequential estimation scheme (Lorenc et al. 1991), but with modifications specific to Mars (Lewis et al. 2007). 

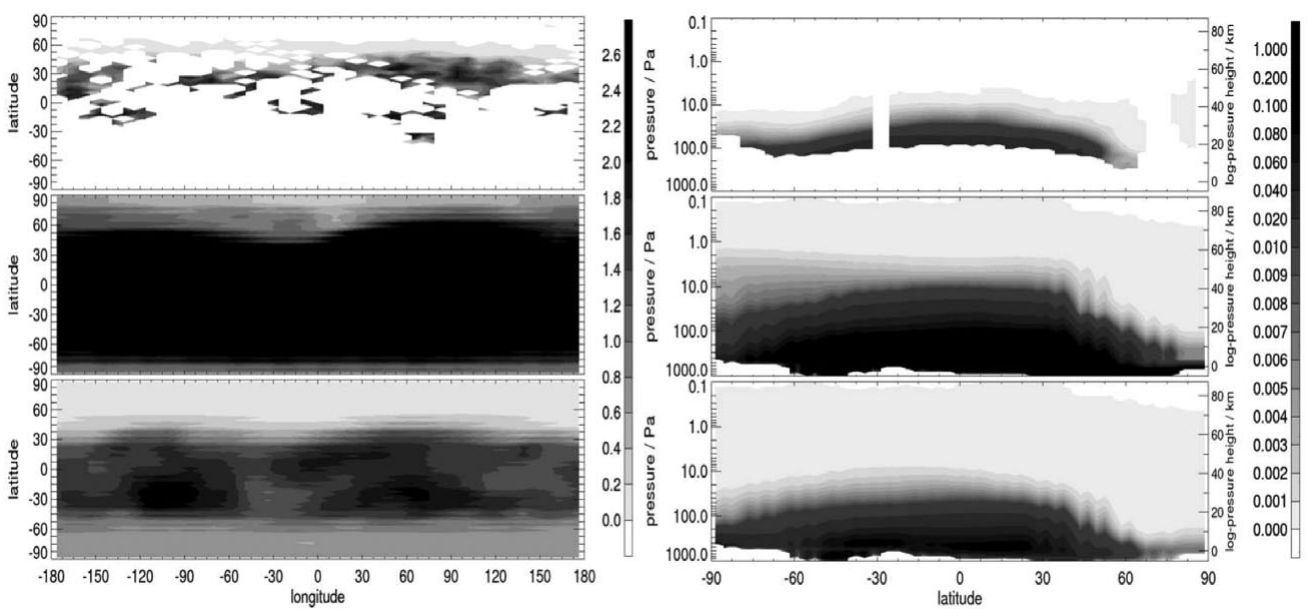

Figure 1. Comparisons among observations (top panels in each column), free-running simulations (middle panels in each column) and assimilations (bottom panels in each column) in night time average fields (between local time 18:00 and 06:00). The left column shows the column dust opacity at the reference level $(610 \mathrm{~Pa})$, and the right column presents the zonal average of dust opacity $\left(\mathrm{km}^{-1}\right)$ at each retrieved level. All values are averaged in a $5^{\circ}$ solar longitude $\left(L_{s}\right)$ time window (centered at $L_{s}=307.5^{\circ}$ in MY28, the period when the global dust storm started to dissipate) and interpolated onto the same horizontal grid and vertical levels as the retrievals.

\section{Earlier studies}

In our earlier studies, the assimilation of temperature profiles and column dust opacity without dust transport has been used to investigate a number of climatological phenomena in the Martian atmosphere.

\section{Atmospheric impacts of global dust storms:}

Montabone et al. 2005 used this assimilated record to study the atmospheric impacts of global dust storms. The assimilation indicated the dust storm evolved from a regional scale to encircle the whole planet in Martian Year (MY) 25, and lasted for a relatively long period. High dust loadings during the MY25 global dust storm also had significant impacts on the thermal structure of the atmosphere.

\section{Super rotating jets with high dust loading:}

Idealized standalone Mars model simulations have been conducted to investigate the formation of tidally-forced, super-rotating jets under Mars-like conditions (Lewis \& Read 2003). In order to represent the super-rotation quantitatively, the local super-rotation index $(s)$ was defined in the work of Read (1986) as the excess zonal-mean angular momentum about the rotation axis, normalized by the angular momentum of air at rest at the equator. The idealized model studies suggested that the higher dust loadings would lead to larger super-rotation index by enhancing the thermal tide. The assimilated records showed that a larger super-rotation index occurred during the MY25 global dust storm, consistent with these idealized models (Lewis et al. 2012).

\section{Improved data assimilation system}

An earlier attempt at Martian data assimilation (e.g. Lewis et al. 2007) focused on assimilation of thermal profiles, with total column dust opacity assimilation found to be important. Dust transport was not active within this version of the model since there was no information from TES data about the dust vertical distribution. In the present 


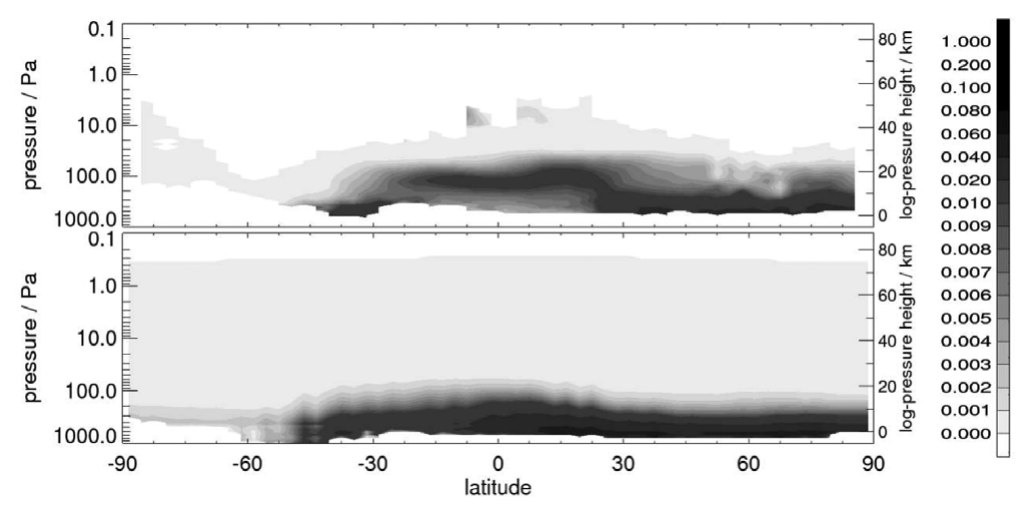

Figure 2. The local zonal mean dust opacity $\left(\mathrm{km}^{-1}\right)$ at each retrieved level using MCS data (upper panel for observation, and lower for assimilation of total dust opacity) at $L_{s}=117.5^{\circ}$. The free-running MGCM also missed this feature, but results were not shown here.

work, full dust transport has been activated while correcting total column dust opacity. A comparison with observations shows an improved simulation of the top of the dust layer as a function of position. Although most of the dust observations could not be retrieved during global dust storms, the assimilation with retrievals available after the peak of a global dust storm obtained a more realistic dissipation of this global dust storm (left column of Fig. 1), as well as a better shape of the dust layer's top, which was higher at lower latitudes but descended when approaching the south pole (right column of Fig. 1). Moreover, the retreat of the global dust storm from the northern polar region was captured better in this new assimilation. However, both assimilations of total dust opacity data and free-running simulations seem to fail to capture the observed detached dust layers at low latitudes (Fig. 2).

\section{Discussion \& future work}

The more advanced data assimilation system has produced reasonable records of dust storm evolution. However, the inability to capture detached dust layers during the Northern spring and summer will require a more detailed understanding of dust processes to improve the representation of the dust cycle. Assimilation of complete dust vertical profiles with fully active dust transport will soon be implemented into the MGCM, and it is hoped this will improve the simulation of the detached dust layers. It also provides a better opportunity to investigate the initialization and vertical structure of global dust storms.

\section{References}

Lewis, S. R. \& Read, P. L. 2003, J. Geophys. Res., 108, 5034

Lewis, S. R., Read, P. L., Conrath, B. J. Pearl, J. C., \& Smith M. D. 2007, Icarus, 192, 327

Lewis, S. R., Read, P. L. Ruan, T., \& Montabone, L. 2012, EPSC Abstract, 7

Lorenc, A. C., Bell, R. S., \& Macpherson, B. 1991, Q. J. R. Meteor. Soc., 117, 59

McCleese, D. J., Schofield, J. T., Taylor, F. W., Calcutt, S. B., Foote, M. C., Kass, D. M., Leovy, C. B., Paige, D. A., Read, P. L., \& Zurek, R. W. 2007, J. Geophys. Res., 112, E05S06

Montabone, L., Lewis, S. R., \& Read, P. L. 2005, Adv. Space Res., 36, 2146

Read, P. L. 1986, Q. J. R. Meteor. Soc., 112, 253

Smith, M. D. 2004, Icarus, 167, 148 